\title{
Review Article \\ Serum Uric Acid Increases Risk of Cancer Incidence and Mortality: A Systematic Review and Meta-Analysis
}

\author{
Shushan Yan, ${ }^{1,2}$ Pengjun Zhang, ${ }^{3}$ Wei Xu, ${ }^{4}$ Yuqing Liu, ${ }^{5}$ Bin Wang, ${ }^{6}$ Tao Jiang, \\ Changjiang Hua, ${ }^{1}$ Xuan Wang, ${ }^{1}$ Donghua $X u,{ }^{7}$ and Beicheng Sun ${ }^{2}$ \\ ${ }^{1}$ Department of Surgical Oncology, The Eighty-First Hospital of People's Liberation Army, Nanjing 210002, China \\ ${ }^{2}$ Liver Transplantation Center of the First Affiliated Hospital, Nanjing Medical University, Nanjing 210029, China \\ ${ }^{3}$ Department of Surgery Emergency, People's Hospital of Rizhao, Rizhao 276800, China \\ ${ }^{4}$ Center of Computer Network, People's Hospital of Rizhao, Rizhao 276800, China \\ ${ }^{5}$ Department of Cardiology, People's Hospital of Rizhao, Rizhao 276800, China \\ ${ }^{6}$ Department of Endocrinology, The Affiliated Hospital of Medical College, Qingdao University, Qingdao 266003, China \\ ${ }^{7}$ Department of Rheumatism, The First Affiliated Hospital, Nanjing Medical University, Nanjing 210029, China
}

\begin{abstract}
Correspondence should be addressed to Shushan Yan; shushanyan_doc@sina.com, Pengjun Zhang; zhangpengjun226@163.com
\end{abstract} and Beicheng Sun; beichengsun_doc@aliyun.com

Received 8 October 2014; Accepted 1 December 2014

Academic Editor: Anshu Agrawal

Copyright (C) 2015 Shushan Yan et al. This is an open access article distributed under the Creative Commons Attribution License, which permits unrestricted use, distribution, and reproduction in any medium, provided the original work is properly cited.

\begin{abstract}
SUA is a potent antioxidant and thus may play a protective role against cancer. Many epidemiological studies have investigated this hypothesis but provided inconsistent and inconclusive findings. We aimed to precisely elucidate the association between SUA levels and cancer by pooling all available publications. Totally, 5 independent studies with 456,053 subjects and 12 with 632,472 subjects were identified after a comprehensive literature screening from PubMed, Embase, and Web of Science. The pooled RRs showed that individuals with high SUA levels were at an increased risk of total cancer incidence (RR $=1.03,95 \%$ CI $1.01-1.05, P=0.007)$. Positive association between high SUA levels and total cancer incidence was observed in males but not females (for men: RR $=1.05$, 95\% CI 1.02-1.08, $P=0.002$; for women, RR $=1.01$, 95\% CI 0.98-1.04, $P=0.512$ ). Besides, high SUA levels were associated with an elevated risk of total cancer mortality $(\mathrm{RR}=1.17,95 \% \mathrm{CI} 1.04-1.32, P=0.010)$, particularly in females $(\mathrm{RR}=1.25,95 \% \mathrm{CI}$ $1.07-1.45, P=0.004)$. The study suggests that high SUA levels increase the risk of total cancer incidence and mortality. The data do not support the hypothesis of a protective role of SUA in cancer.
\end{abstract}

\section{Introduction}

Serum uric acid (SUA) is one of the most abundant molecules with antioxidant properties in human blood acting as a free radical scavenger and a chelator of transitional metal ion $[1,2]$. However, increased SUA is a highly prevalent condition with controversial health consequences. Emerging data has suggested the causative role of elevated UA levels in cardiovascular, respiratory diseases, renal diseases, and metabolic syndrome [2-4]. Hyperuricemia is a consequence of impaired kidney function and can increase the risk of chronic kidney disease and end-stage renal disease [5]. Low levels of SUA are detrimental to the neurons, while high levels of SUA contribute to inflammation and neuroprotection [6].
Interestingly, it has been hypothesized that SUA may confer protective effects on cancer due to its antioxidant property [7]. However, currently published epidemiological studies on the association between SUA levels and cancerrelated incidence and mortality have provided conflicting and inconclusive findings possibly because of different study design, source of controls, sample size, and statistical power. The study by Kuo et al. has suggested that low SUA levels are associated with elevated risk of cancer-related mortality compared with high SUA levels, which implicates a protective role of SUA in cancer [8]. On the contrary, Strasak AM and colleagues have demonstrated that high SUA levels are independently related to increased risk of total cancer mortality [9]. To shed light on understanding the paradoxical 
TABLE 1: Characteristics of all studies.

\begin{tabular}{lcccccccc}
\hline First author & Year & \multicolumn{1}{c}{ Study design } & Origins & Sample size & Followup (years) & Baseline time & Sex & Cancer \\
\hline Cancer incidence & & & & & & & & \\
Horsfall [4] & 2014 & Prospective cohort study & UK & 205,484 & 5 & $2000-2012$ & Mixed & Lung \\
Strasak [10] & 2009 & Prospective cohort study & Austrian & 78,850 & 12.4 & $1985-2003$ & Men & All \\
Kolonel [11] & 1994 & Prospective cohort study & Hawaii & 7,889 & 27 & $1965-1968$ & Men & All \\
Hiatt [12] & 1988 & Prospective cohort study & USA & 75,283 & 9.8 & $1965-1972$ & Men & All \\
Hiatt [12] & 1988 & Prospective cohort study & USA & 88,547 & 9.8 & $1965-1972$ & Women & All \\
Cancer mortality & & & & & & & & Men \\
Taghizadeh [13] & 2014 & Prospective cohort study & Dutch & 4,350 & 38 & $1965-1969$ & All \\
Juraschek [14] & 2014 & Prospective cohort study & Scotland & 15,083 & 22.7 & $1984-1987$ & Mixed & All \\
Kuo [8] & 2013 & Prospective cohort study & Taiwan & 354,110 & 9 & $2000-2007$ & Mixed & All \\
Strasak [15] & 2007 & Prospective cohort study & Austrian & 28,613 & 15.2 & $1985-2005$ & Women & All \\
Strasak [15] & 2007 & Prospective cohort study & Austrian & 83,683 & 13.6 & $1985-2005$ & Men & All \\
Jee [16] & 2004 & Prospective cohort study & Korean & 22,698 & 9 & $1992-1996$ & Men & All \\
Colangelo [3] & 2002 & Prospective cohort study & USA & 20,433 & 26.2 & $1967-1973$ & Men & Colorectal cancer \\
Colangelo [3] & 2002 & Prospective cohort study & USA & 15,149 & 26.2 & $1967-1973$ & Women & Colorectal cancer \\
Tomita [17] & 2000 & Prospective cohort study & Japanese & 49,413 & 5.4 & $1975-1982$ & Men & All \\
Gapstur [18] & 2000 & Prospective cohort study & USA & 20,475 & 25 & $1963-1973$ & Men & Pancreatic cancer \\
Gapstur [18] & 2000 & Prospective cohort study & USA & 15,183 & 25 & $1963-1973$ & Women & Pancreatic cancer \\
Mazza [19] & 1999 & Prospective cohort study & Italy & 3,282 & 12 & Not reported & Mixed & All \\
\hline
\end{tabular}

role of SUA in the risk of cancer incidence and mortality, we performed this meta-analysis of all currently published studies.

\section{Materials and Methods}

2.1. Search Strategy. A comprehensive literature screening from databases of PubMed, Embase, and Web of Science was performed for eligible studies from their inception up to August 12, 2014. We used the following terms: uric acid, serum uric acid, gout, or hyperuricemia; cancer, tumor, or carcinoma; cancer risk, cancer incidence, or cancer mortality. The references of all retrieved studies were also screened for additional papers. There were no language restrictions for literature search.

2.2. Inclusion and Exclusion Criteria. Studies were included in our study if they conform to the following inclusion criteria: (1) studies in cohort designs; (2) studies on the relationship between SUA and cancer; (3) studies with data of odds ratio (ORs), relative risks (RRs), or hazard ratios (HRs) with $95 \%$ confidence intervals (95\% CIs). Studies not related to the risk of cancer incidence and mortality, case reports, reviews, animal studies, and studies with overlapping data were all excluded.

2.3. Data Extraction. Data were extracted by two investigators independently. Disagreements were resolved by consensus. Relevant data were as follows: first author, year of publication, origins, study designs, specific sites of cancer, cancer types, sex, age, sample size, baseline time, followup duration, adjusted factors, and RRs or HRs or ORs with corresponding 95\% CIs for the risk of cancer incidence and mortality. Available RRs or HRs or ORs with 95\% CIs were extracted based on the highest SUA levels in each included study.

2.4. Statistical Analysis. We evaluated the strength for association between SUA levels and cancer by calculating the pooled RRs with 95\% CIs. Cochran's Q-statistic test and $I^{2}$ test were performed to estimate the between-study heterogeneity, and $P<0.05$ and $I^{2}>50 \%$ suggested potential heterogeneity across all studies [20, 21]. The fixed-effects model by MantelHaenszel method was applied when the between-study heterogeneity was insignificant [22]; otherwise, the randomeffects model by DerSimonian and Laird method was used [23]. Stratified analyses by sex and specific sites of cancer were also carried out. Sensitivity analysis was performed to assess the influence of single studies. Begg's funnel plots and Egger's test were adopted to estimate publication bias risk [24, 25]. STATA 12.0 software (StataCorp, College Station, TX, USA) was used for all analyses.

\section{Results}

3.1. Literature Search and Characteristics of All Studies. We performed a comprehensive literature search in PubMed, Embase, and Web of Science databases for eligible studies. According to the inclusion criteria, 5 independent studies on cancer incidence with 456,053 subjects $[4,10-12]$ and 12 on cancer mortality with 632,472 subjects [3, 8, 9, 13-19] were finally included in our study. All included studies were in prospective cohort designs, which were published between 1999 and 2014 (Table 1). Other characteristics including first author, year of publication, sample size, follow-up duration, baseline time, sex, and cancer type were presented in Table 1 at length. The study by Hiatt and Fireman estimated roles 
TABLE 2: Summary meta-analysis results for the association between SUA levels and cancer.

\begin{tabular}{|c|c|c|c|c|c|}
\hline Group/subgroup & Number & $\mathrm{RR}(95 \% \mathrm{CI})$ & $\begin{array}{l}P \text { value for } \\
\text { pooled } \\
\text { analysis }\end{array}$ & $I^{2}(\%)$ & $\begin{array}{c}P \text { value for } \\
\text { heterogeneity } \\
\text { analysis }\end{array}$ \\
\hline \multicolumn{6}{|l|}{ Cancer incidence } \\
\hline All & 456,053 & $1.03(1.01-1.05)$ & 0.007 & 44.7 & 0.124 \\
\hline Men & 162,022 & $1.05(1.02-1.08)$ & 0.002 & 53.8 & 0.115 \\
\hline Women & 88,547 & $1.01(0.98-1.04)$ & 0.512 & - & - \\
\hline \multicolumn{6}{|l|}{ Specific sites } \\
\hline Any & 250,569 & $1.03(1.01-1.05)$ & 0.006 & 57.9 & 0.068 \\
\hline Respiratory system and intrathoracic organs & 456,053 & $1.05(0.93-1.19)$ & 0.448 & 67.5 & 0.015 \\
\hline Digestive organs & 266,347 & $1.08(0.94-1.25)$ & 0.263 & 63.4 & 0.018 \\
\hline Urinary organs & 86,739 & $1.17(0.44-3.15)$ & 0.752 & 82.3 & 0.018 \\
\hline Lymphoid and hematopoietic systems & 86,739 & $1.71(1.10-2.68)$ & 0.018 & 30.0 & 0.232 \\
\hline Male genital organs & 162,022 & $1.06(1.00-1.13)$ & 0.058 & 56.1 & 0.103 \\
\hline \multicolumn{6}{|l|}{ Cancer mortality } \\
\hline All & 632,472 & $1.17(1.04-1.32)$ & 0.010 & 65.8 & 0.001 \\
\hline Men & 201,052 & $1.13(0.86-1.48)$ & 0.384 & 77.5 & $<0.001$ \\
\hline Women & 58,945 & $1.25(1.07-1.45)$ & 0.004 & 30.0 & 0.240 \\
\hline \multicolumn{6}{|l|}{ Specific sites } \\
\hline Any & 561,232 & $1.17(1.04-1.32)$ & 0.011 & 70.7 & 0.001 \\
\hline Respiratory system and intrathoracic organs & 116,646 & $1.08(0.61-1.91)$ & 0.786 & 78.2 & 0.010 \\
\hline Digestive organs & 187,886 & $1.27(1.08-1.49)$ & 0.003 & 40.1 & 0.124 \\
\hline Urinary organs & 112,296 & $1.35(0.88-2.07)$ & 0.172 & 0.0 & 0.681 \\
\hline Lymphoid and hematopoietic systems & 112,296 & $1.18(0.82-1.70)$ & 0.364 & 0.0 & 0.837 \\
\hline Bone, connective tissue, soft tissue, and skin & 112,296 & $0.94(0.47-1.87)$ & 0.857 & 0.0 & 0.588 \\
\hline Male genital organs & 88,033 & $0.51(0.07-3.85)$ & 0.516 & 75.5 & 0.044 \\
\hline
\end{tabular}

of SUA in cancer incidence among males and females, respectively, and thus it was regarded as two independent studies [12]. Based on different gender, two other papers on the association between SUA and cancer mortality were also divided into two individual studies, respectively $[3,18]$.

3.2. Association between SUA and the Risk of Cancer Incidence. We found that high SUA levels were associated with an increased risk of total cancer incidence by meta-analysis of 5 independent studies $(\mathrm{RR}=1.03,95 \% \mathrm{CI} 1.01-1.05, P=0.007)$ (Table 2, Figure 1(a)).

When stratifying analysis by sex, the pooled RRs showed that high SUA levels were significantly related to the risk of cancer incidence among males but not females (for men: RR $=1.05,95 \%$ CI 1.02-1.08, $P=0.002$; for women, $\mathrm{RR}=1.01$, 95\% CI 0.98-1.04, $P=0.512$ ) (Table 2, Figure 1(a)). However, there was only one relevant study on the cancer incidence risk in relation to SUA among females.

When stratifying analysis by specific sites of cancer, significant relationship between high SUA levels and the risk of lymphoid and hematopoietic system cancers was observed, but not other specific sites of cancer (Table 2). Sensitivity analysis did not materially alter the pooled results mentioned above.
3.3. Association between SUA and the Risk of Cancer Mortality. The pooled RRs revealed that individuals with high SUA levels were at an elevated risk of total cancer mortality (RR $=1.17,95 \%$ CI 1.04-1.32, $P=0.010$ ) (Table 2, Figure 1(b)). Such significant association was demonstrated in females rather than males (for females: $\mathrm{RR}=1.25$, 95\% CI 1.07$1.45, P=0.004$; for males: $\mathrm{RR}=1.13$, 95\% CI $0.86-$ $1.48, P=0.384$ ) (Table 2, Figure 1(b)). The between-study heterogeneity was not significant in studies performed among women. Thus, fixed-effects model was used to estimate the role of SUA in cancer mortality among females (Table 2). The combined results were further confirmed by sensitivity analysis.

In stratifying analysis by specific sites of cancer, high SUA levels were found to correlate with an elevated risk of digestive cancers mortality $(\mathrm{RR}=1.27,95 \%$ CI $1.08-1.49, P=0.003$ ) (Table 2). No significant association was observed in relation to the mortality of other cancers (Table 2). Sensitivity analysis did not modify the pooled results.

3.4. Publication Bias. No potential publication bias risk was found in our study, as suggested by both Begg's funnel plots and Egger's test (Figures 1(c) and 1(d)). Similar findings were demonstrated in both studies related to cancer incidence and studies associated with cancer mortality. 


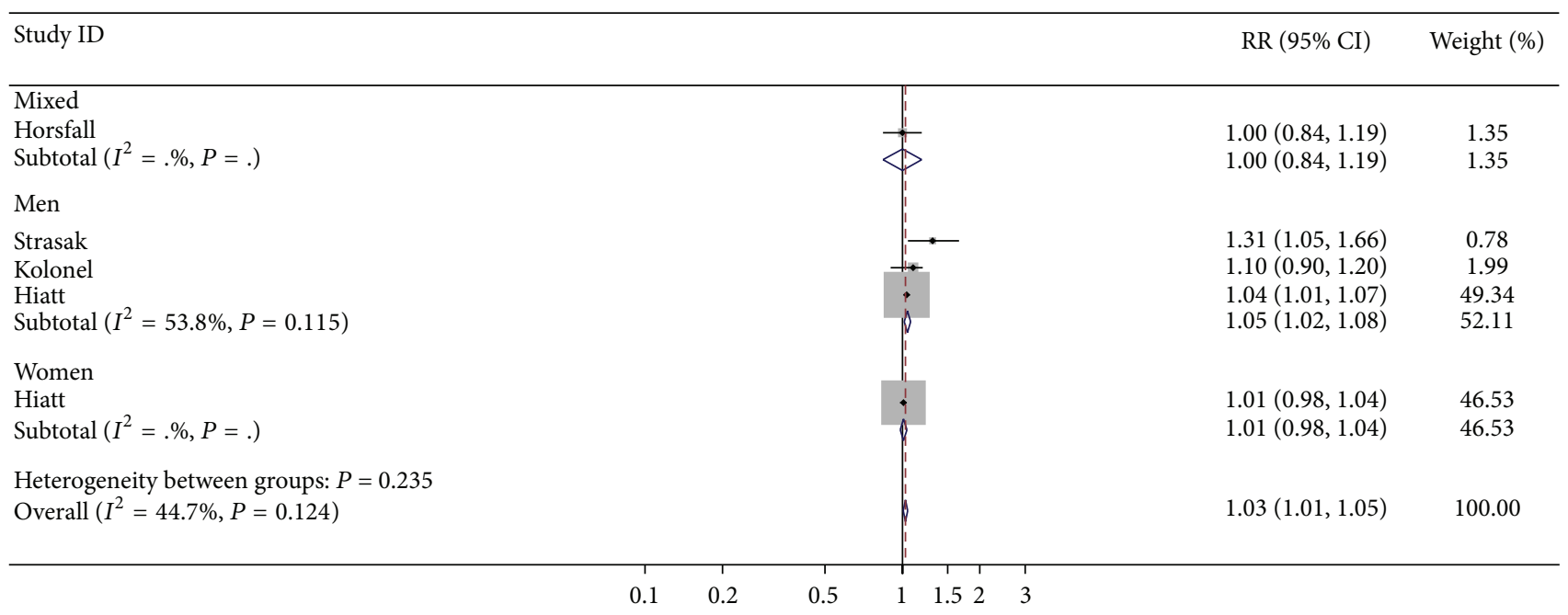

(a)

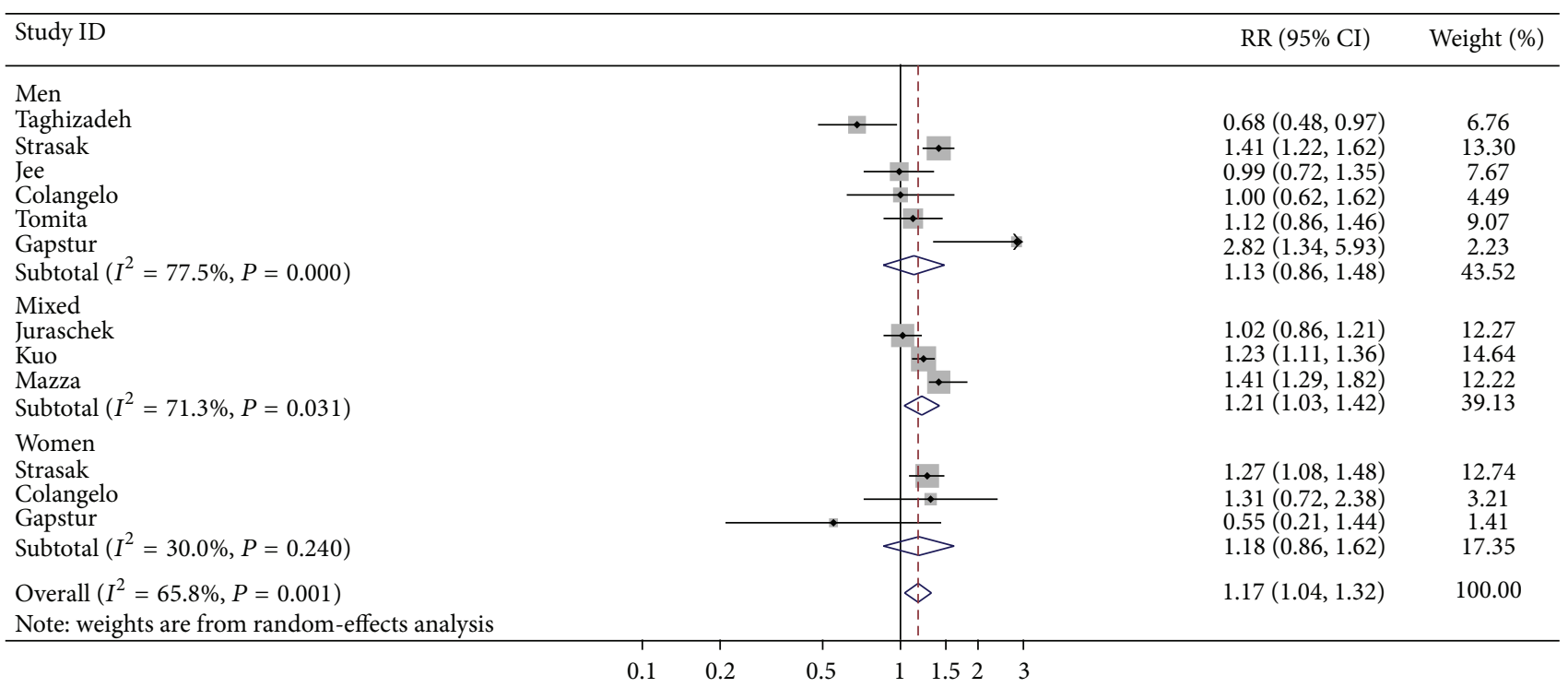

(b)

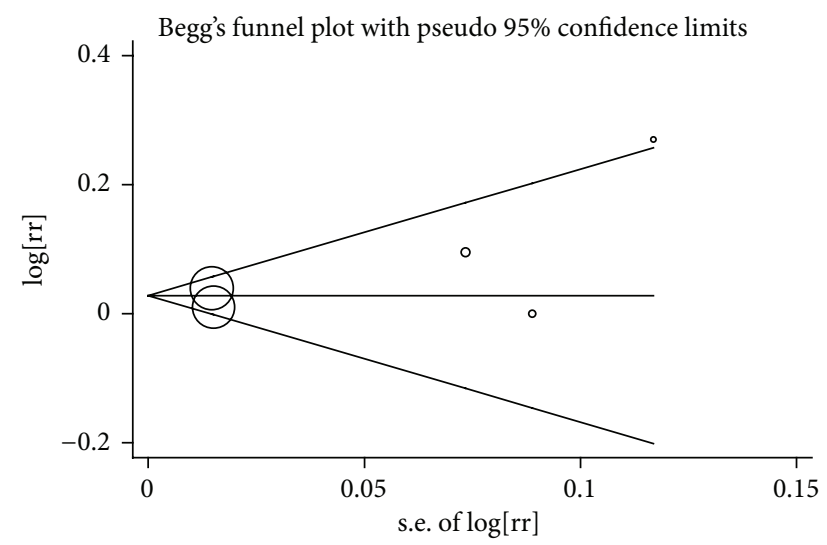

(c)

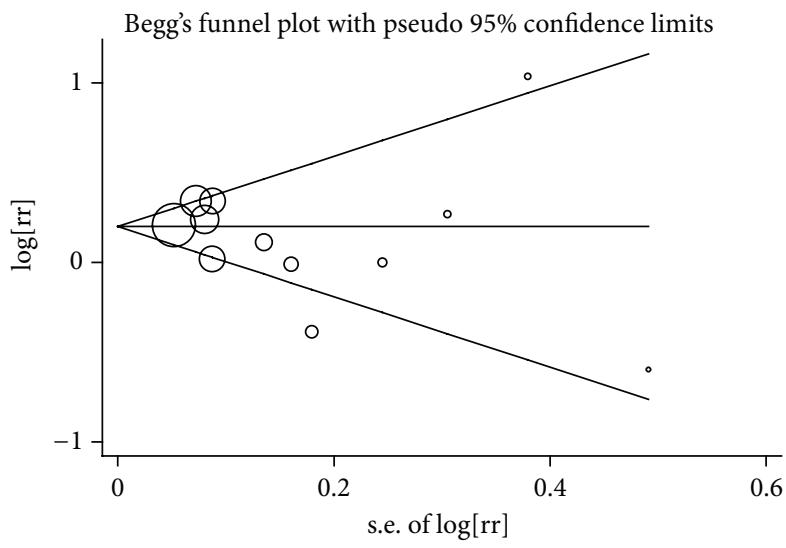

(d)

FIGURE 1: (a) Forest plot for SUA and the risk of cancer incidence; (b) forest plot for SUA and the risk of cancer mortality; (c) Begg's funnel plots for the risk of cancer incidence ( $P$ value for Egger's test equal to 0.295); (d) Begg's funnel plots for the risk of cancer mortality $(P$ value for Egger's test equal to 0.354). 


\section{Discussion}

The current meta-analysis shows the evidence that high SUA levels are associated with increased risk of cancer incidence and mortality. The association between SUA and the risk of cancer-related incidence and mortality differs by gender. Positive association between high SUA levels and total cancer incidence was observed in males but not females. Besides, high SUA levels were associated with an elevated risk of total cancer mortality in females but not males.

To the best of our knowledge, UA is produced from metabolic conversion of either dietary or endogenous purines. The primary sites of excretion of UA are kidney and gut. UA is capable of scavenging free radical and chelating transitional metal ions by preventing peroxynitriteinduced protein nitrosylation, lipid and protein peroxidation, and the inactivation of tetrahydrobiopterin [2]. Nonetheless, studies have also suggested a prooxidant role of UA [2]. Elevated levels of SUA can enhance inflammatory response and contribute to hyperuricemia, gout, cardiovascular, and renal complications. It is particularly interesting since UA plays dual roles as antioxidant and prooxidant. Concerning malignant diseases, SUA has drawn much attention for the past few decades. High cell turnover and tumor lysis syndrome in certain cancers are responsible for increased levels of UA in human serum, implicating potential link between SUA and cancer $[8,26,27]$. It has been demonstrated that UA can provide an antioxidant defense against oxidantand radical-caused aging and cancer [7]. Nevertheless, UA has also been reported as an independent risk factor for cancer incidence and mortality in other studies $[9,28]$. Thus, the precise relationship of SUA with cancer remains obscure and needs further elucidation.

Tobacco smoking is a well-established risk factor of lung cancer. Horsfall and colleagues have found that current smokers with low levels of SUA are more susceptible to lung cancer, which suggests a protective role of SUA and an interaction between SUA and smoking in lung carcinogenesis [4]. Reversely, the study by Strasak et al. has shown that elevated levels of SUA are related to higher risk of several site-specific malignancies, for instance, digestive organs, connective tissue, soft tissue and skin, lung cancer, urinary organs, bone, and hematopoietic cancers [10]. However, no significant association between increased SUA levels and the risk of total cancer or cancers of lung, stomach, colon, rectum, bladder, or hematopoietic system was demonstrated by Kolonel et al. [11]. The contradictory findings may be attributed to diverse methodology, ethnicity, environmental exposures, source of controls, and statistical power. Metaanalysis with large sample size is more powerful in identifying minor association by pooling all currently available publications. A total of 5 independent studies on cancer incidence and 12 on cancer mortality were included in the present metaanalysis. The pooled results suggested that elevated SUA levels were positively associated with the risk of total cancer and lymphoid and hematopoietic system cancers, implicating a risk role of SUA in carcinogenesis. Moreover, gender bias was observed when estimating the relationship between SUA levels and cancer risk, which warranted elucidation in more relevant cohort studies with large sample size.

The effect of SUA levels on cancer mortality seems inconsistent. Taghizadeh et al. reported that higher levels of SUA were related to lower risk of cancer mortality, which supported a protective role of SUA in the risk of cancer mortality [13]. Nonetheless, elevated SUA levels were demonstrated to be an independent risk factor for cancerrelated mortality in several other cohort studies $[9,14,19]$. To shed some light on the controversial findings, we estimated the association between SUA and the risk of cancer mortality by meta-analysis of all currently available data. Statistically significant association was observed between higher SUA levels and increased mortality of total cancer and digestive cancers. Besides, the positive correlation was more significant in females than males. Different amounts of SUA, saturation of UA levels in cancer, and metabolism of SUA may be responsible for the risk discrepancy between women and men with cancer. Interestingly, the significant association was related to digestive cancers, but not other specific sites of cancer, implicating site bias in the relationship between higher SUA levels and cancer mortality. The statistically significant association may be a chance finding in that only one eligible study was included related to the mortality of pancreatic cancer and colorectal cancer, respectively $[3,18]$. In addition, the inconsistent findings for diverse specific sites of cancer might be attributed to variable number of years for cancer mortality, different study design, sample size, environmental exposures, and genetic background. More studies with high quality are warranted to provide a precise estimate for the role of SUA in cancer-related mortality, particularly with regard to specific sites of malignancies and gender.

\section{Limitations}

The pooled results should be interpreted with caution due to some limitations in our study. First, significant gender bias was found when assessing the effect of SUA on the risk of cancer incidence and mortality; however, the sample size in each stratified analysis by sex seemed a bit limited. More relevant studies with sufficient statistical power are recommended for further elucidation. Second, a significant interaction between SUA and smoking was demonstrated in the development of cancer [4]. We failed to perform stratified analysis by smoking status for lack of enough available studies published to date. It can be further investigated in more future studies. Last but not least, although no significant publication bias was observed in our study, potential bias might be introduced in that not all included studies were based on the same adjusted factors, such as age, gender, environmental exposures, and ethnicity. Thus, the findings in this meta-analysis should be interpreted seriously.

\section{Conclusions}

In summary, the present study suggests that elevated SUA levels are related to increased risk of cancer incidence and mortality. However, the precise association between SUA 
levels and cancer warrants further investigation in more independent studies with high quality in the future.

\section{Abbreviations}

$\begin{array}{ll}\text { ORs: } & \text { Odds ratios } \\ \text { RRs: } & \text { Relative risks } \\ \text { HRs: } & \text { Hazard ratios } \\ \text { 95\% CIs: } & \text { 95\% confidence intervals } \\ \text { SUA: } & \text { Serum uric acid. }\end{array}$

\section{Conflict of Interests}

The authors declare that there is no conflict of interests regarding the publication of this paper.

\section{Authors' Contribution}

Shushan Yan, Pengjun Zhang, and Beicheng Sun conceived and designed the experiments. Bin Wang, Wei $\mathrm{Xu}$, Yuqing Liu, and Tao Jiang performed the experiments and collected data. Bin Wang and Xuan Wang analyzed the data. Changjiang Hua and Xuan Wang contributed reagents/materials/analysis tools. Shushan Yan, Pengjun Zhang, Beicheng Sun, and Donghua Xu wrote the paper.

\section{References}

[1] G. K. Glantzounis, E. C. Tsimoyiannis, A. M. Kappas, and D. A. Galaris, "Uric acid and oxidative stress," Current Pharmaceutical Design, vol. 11, no. 32, pp. 4145-4151, 2005.

[2] D. H. Kang and S. K. Ha, "Uric acid puzzle: dual role as antioxidantand pro-oxidant," Electrolyte and Blood Pressure, vol. 12, no. 1, pp. 1-6, 2014.

[3] L. A. Colangelo, S. M. Gapstur, P. H. Gann, A. R. Dyer, and K. Liu, "Colorectal cancer mortality and factors related to the insulin resistance syndrome," Cancer Epidemiology Biomarkers and Prevention, vol. 11, no. 4, pp. 385-391, 2002.

[4] L. J. Horsfall, I. Nazareth, and I. Petersen, "Serum uric acid and the risk of respiratory disease: a population-based cohort study," Thorax, vol. 69, pp. 1021-1026, 2014.

[5] I. Y. Kim, D. W. Lee, S. B. Lee, and I. S. Kwak, “The role of uric acid in kidney fibrosis: experimental evidences for the causal relationship," BioMed Research International, vol. 2014, Article ID 638732, 9 pages, 2014.

[6] P. Fang, X. Li, J. J. Luo, H. Wang, and X. F. Yang, "A double-edged sword: uric acid and neurological disorders," Brain Disorders \& Therapy, vol. 2, no. 2, p. 109, 2013.

[7] B. N. Ames, R. Cathcart, E. Schwiers, and P. Hochstein, "Uric acid provides an antioxidant defense in humans against oxidant- and radical-caused aging and cancer: a hypothesis," Proceedings of the National Academy of Sciences of the United States of America, vol. 78, no. 11, pp. 6858-6862, 1981.

[8] C.-F. Kuo, L.-C. See, K.-H. Yu, I.-J. Chou, M.-J. Chiou, and S.-F. Luo, "Significance of serum uric acid levels on the risk of allcause and cardiovascular mortality," Rheumatology, vol. 52, no. 1, pp. 127-134, 2013.

[9] A. M. Strasak, K. Rapp, W. Hilbe et al., "Serum uric acid and risk of cancer mortality in a large prospective male cohort," Cancer Causes and Control, vol. 18, no. 9, pp. 1021-1029, 2007.
[10] A. M. Strasak, S. Lang, T. Kneib et al., "Use of penalized splines in extended Cox-type additive hazard regression to flexibly estimate the effect of time-varying serum uric acid on risk of cancer incidence: a prospective, population-based study in 78,850 men," Annals of Epidemiology, vol. 19, no. 1, pp. 15-24, 2009.

[11] L. N. Kolonel, C. Yoshizawa, A. M. Y. Nomura, and G. N. Stemmermann, "Relationship of serum uric acid to cancer occurrence in a prospective male cohort," Cancer Epidemiology Biomarkers and Prevention, vol. 3, no. 3, pp. 225-228, 1994.

[12] R. A. Hiatt and B. H. Fireman, "Serum uric acid unrelated to cancer incidence in humans," Cancer Research, vol. 48, no. 10, pp. 2916-2918, 1988.

[13] N. Taghizadeh, J. M. Vonk, and H. M. Boezen, "Serum uric acid levels and cancer mortality risk among males in a large general population-based cohort study," Cancer Causes \& Control, vol. 25, no. 8, pp. 1075-1080, 2014.

[14] S. P. Juraschek, H. Tunstall-Pedoe, and M. Woodward, "Serum uric acid and the risk of mortality during 23 years follow-up in the Scottish heart health extended cohort study," Atherosclerosis, vol. 233, no. 2, pp. 623-629, 2014.

[15] A. M. Strasak, K. Rapp, W. Hilbe et al., "The role of serum uric acid as an antioxidant protecting against cancer: prospective study in more than 28000 older Austrian women," Annals of Oncology, vol. 18, no. 11, pp. 1893-1897, 2007.

[16] S. H. Jee, S. Y. Lee, and M. T. Kim, "Serum uric acid and risk of death from cancer, cardiovascular disease or all causes in men," European Journal of Cardiovascular Prevention and Rehabilitation, vol. 11, no. 3, pp. 185-191, 2004.

[17] M. Tomita, S. Mizuno, H. Yamanaka et al., "Does hyperuricemia affect mortality? A prospective cohort study of Japanese male workers," Journal of Epidemiology, vol. 10, no. 6, pp. 403-409, 2000.

[18] S. M. Gapstur, P. H. Gann, W. Lowe, K. Liu, L. Colangelo, and A. Dyer, "Abnormal glucose metabolism and pancreatic cancer mortality," The Journal of the American Medical Association, vol. 283, no. 19, pp. 2552-2558, 2000.

[19] A. Mazza, E. Casiglia, R. Scarpa et al., "Predictors of cancer mortality in elderly subjects," European Journal of Epidemiology, vol. 15, no. 5, pp. 421-427, 1999.

[20] W. G. Cochran, "The comparison of percentages in matched samples," Biometrika, vol. 37, no. 3-4, pp. 256-266, 1950.

[21] J. P. T. Higgins, S. G. Thompson, J. J. Deeks, and D. G. Altman, "Measuring inconsistency in meta-analyses," British Medical Journal, vol. 327, no. 7414, pp. 557-560, 2003.

[22] N. Mantel and W. Haenszel, "Statistical aspects of the analysis of data from retrospective studies of disease," Journal of the National Cancer Institute, vol. 22, no. 4, pp. 719-748, 1959.

[23] R. DerSimonian and N. Laird, "Meta-analysis in clinical trials," Controlled Clinical Trials, vol. 7, no. 3, pp. 177-188, 1986.

[24] M. Egger, G. D. Smith, M. Schneider, and C. Minder, "Bias in meta-analysis detected by a simple, graphical test," British Medical Journal, vol. 315, no. 7109, pp. 629-634, 1997.

[25] A. E. Stuck, L. Z. Rubenstein, and D. Wieland, "Bias in meta-analysis detected by a simple, graphical test. Asymmetry detected in funnel plot was probably due to true heterogeneity," British Medical Journal, vol. 316, no. 7129, pp. 469-461, 1998.

[26] L. Baeksgaard and J. B. Sørensen, "Acute tumor lysis syndrome in solid tumors-a case report and review of the literature," Cancer Chemotherapy and Pharmacology, vol. 51, no. 3, pp. 187192, 2003. 
[27] A. Sevanian, K. J. A. Davies, and P. Hochstein, "Serum urate as an antioxidant for ascorbic acid," The American Journal of Clinical Nutrition, vol. 54, supplement 6, pp. 1129S-1134S, 1991.

[28] M. A. Fini, A. Elias, R. J. Johnson, and R. M. Wright, "Contribution of uric acid to cancer risk, recurrence, and mortality," Clinical and Translational Medicine, vol. 1, no. 1, p. 16, 2012. 


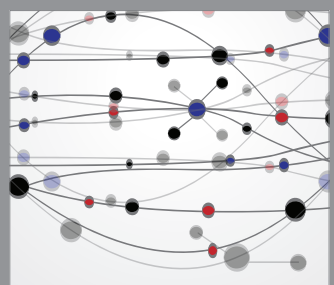

The Scientific World Journal
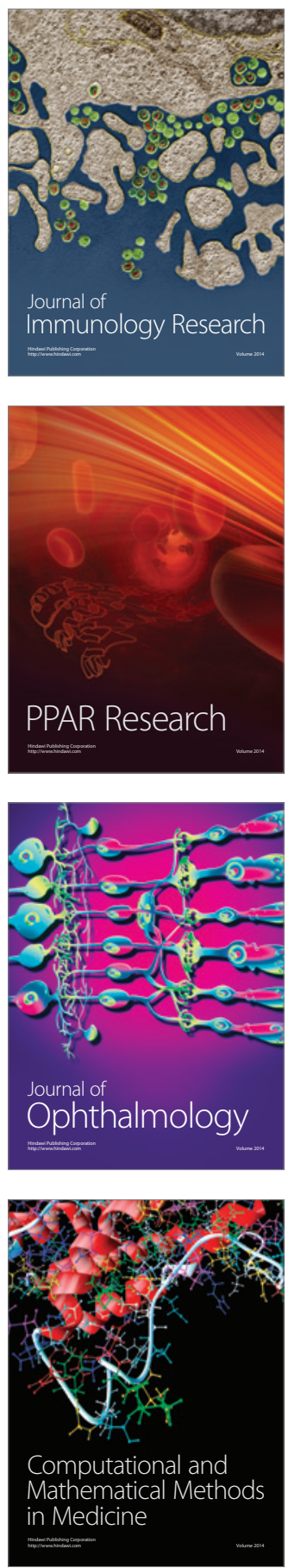

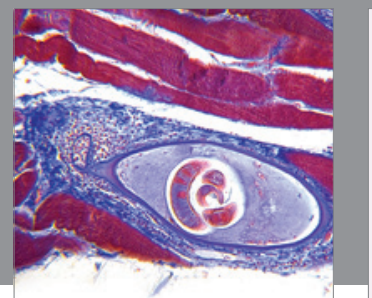

Gastroenterology

Research and Practice
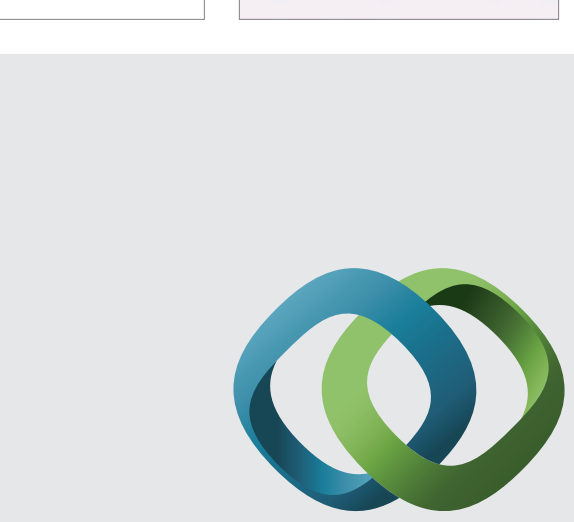

\section{Hindawi}

Submit your manuscripts at

http://www.hindawi.com
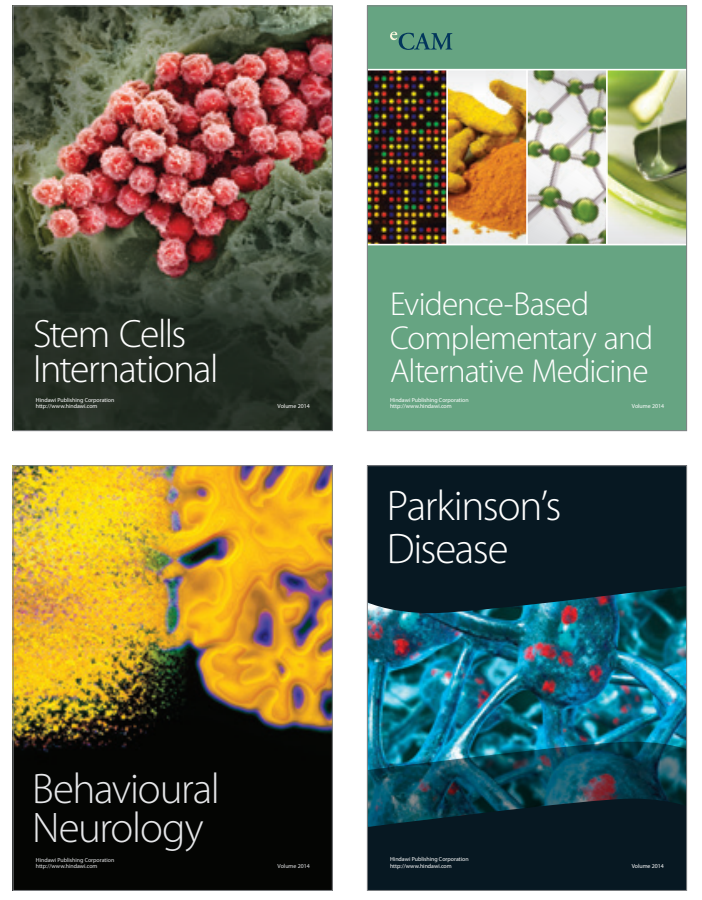
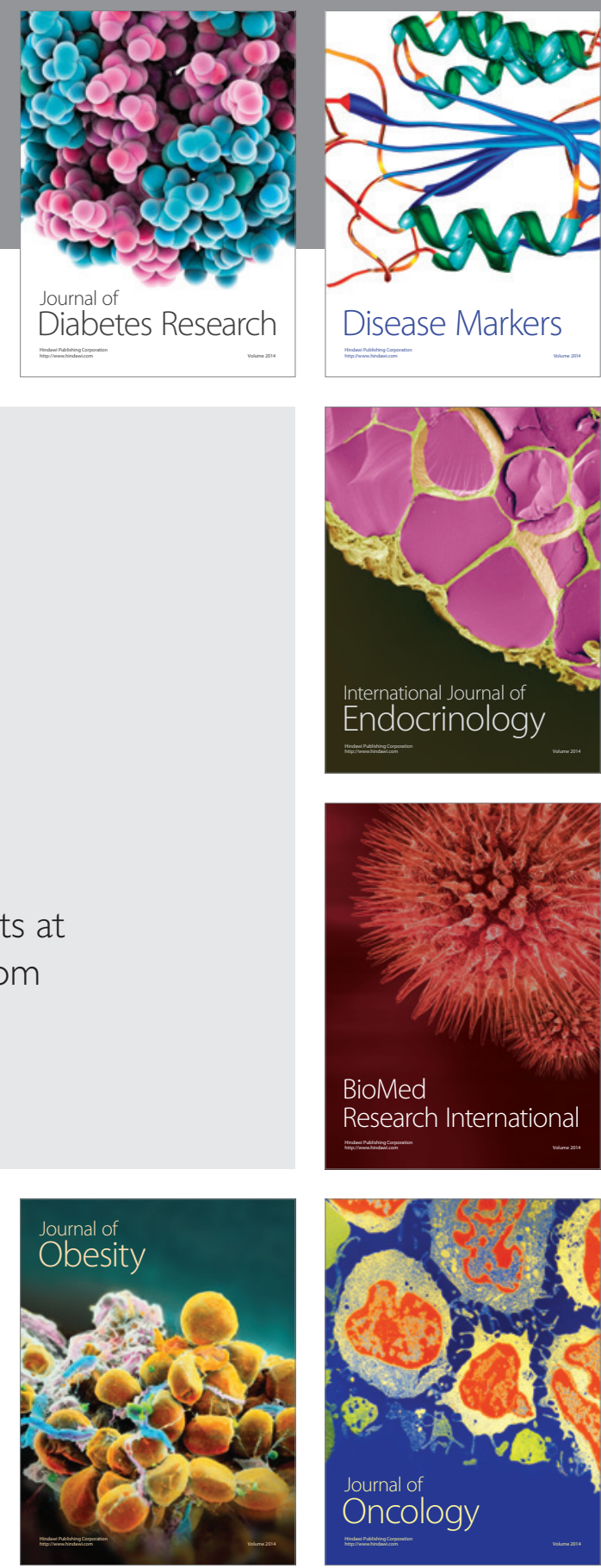

Disease Markers
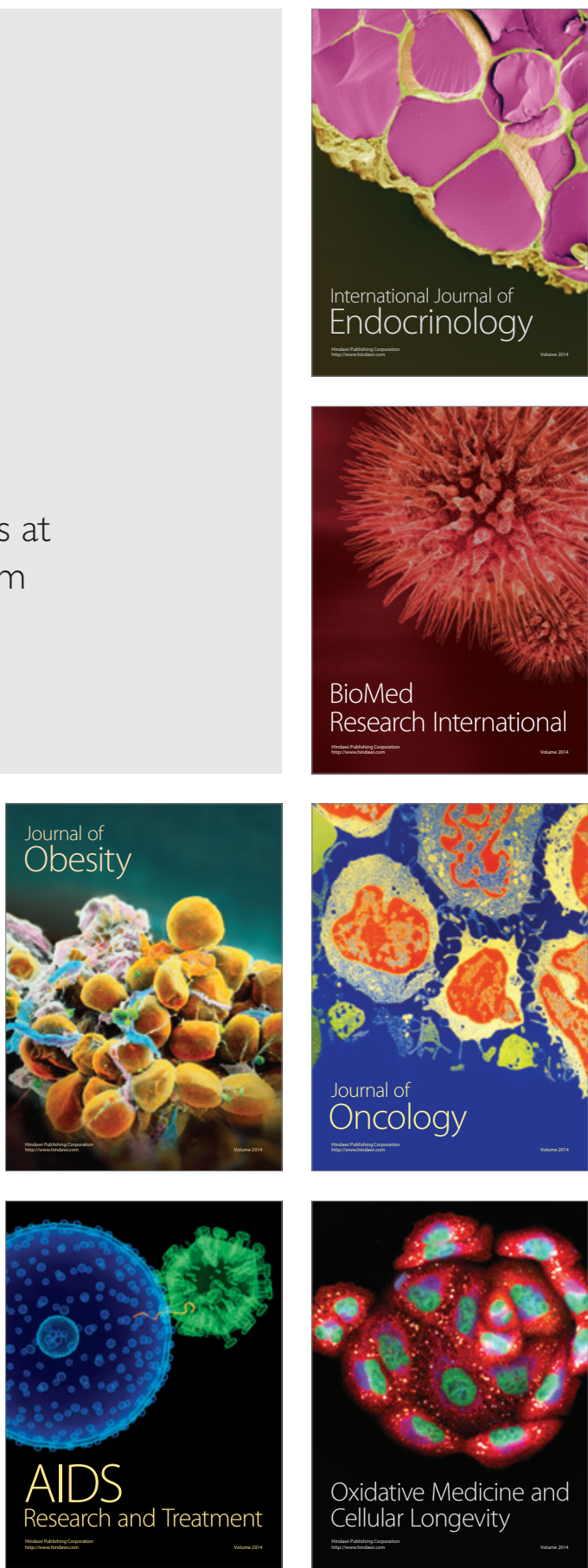